X-RAY BINARIES 


\title{
OBSERVATIONS OF LOW MASS X-RAY BINARIES
}

\author{
ALAN P. SMALE \\ USRA Research Scientist \\ Laboratory for High Energy Astrophysics, Code 668 \\ NASA/GSFC, Greenbelt, MD 20771, USA \\ alan@osiris.gsfc.nasa.gov
}

\begin{abstract}
In this paper I review the properties and behavior of low-mass $\mathrm{X}$-ray binary systems (LMXBs) that contain neutron stars (NS), concentrating on the Galactic bulge sources and bursters. I describe the observed characteristics of LMXBs including their light curves, spectra, eclipses, dips, bursts, flares, pulsations, QPO, long-term periodicities and orbital period changes, and explain how fast timing results and the distinction between ' $\mathrm{Z}$ ' and 'atoll'-type sources provide the key to a unified model of LMXB behavior.
\end{abstract}

\section{Introduction}

A LMXB consists of a neutron star (NS) primary, an accretion disk surrounding the primary in the plane of the orbit, and a late type (F, G, K or M) dwarf (or degenerate dwarf) secondary. The primary can be identified as a NS by direct observations of bursts, pulsations, or derived masses or mass ratios, or by analogy with other sources with similar characteristics. The late-type secondaries can occasionally (i.e. for transients) be observed directly through optical/IR photometry and spectroscopy, but are more often inferred through their derived masses. The duration and properties of $\mathrm{X}$-ray eclipse transitions may also supply supporting evidence for their nature. Late-type secondaries do not have natural winds sufficient to power the $\mathrm{X}$-ray source, and significant mass transfer occurs only when the companion fills its Roche lobe. This mass is passed to the NS through an accretion disk in a way familiar to CV observers. X-ray heating of the accretion disk (and sometimes the companion star) dominates the optical emission. In addition, X-ray heating can evaporate material from the disk, forming an accretion disk corona (ADC) above its central regions. 
In total, there are $\sim 200 \mathrm{X}$-ray binaries known, $\sim 125$ of which are LMXBs. Of these, 48 have known optical (or IR) counterparts, 32 have known orbital periods, 40 are known Type I burst sources, 40 are transients (12 of which burst when active), 17 are found in globular clusters, and 6 are known Z-sources. LMXBs are preferentially located in the Galactic plane, towards the Galactic center, or in clusters, away from regions of star formation. Many LMXBs lie in regions of severe interstellar absorption and have no known counterparts.

Historically there have been many classifications of LMXBs, based on flux level, spectral behavior, etc. Most such classifications can be considered obsolete. Observationally, sources divide into classes based largely on inclination effects and intrinsic luminosity. Physically, recent analysis of fast timing and spectral data show that there is only one really useful classification of LMXBs, based on accretion rate and magnetic field strength:

(i) Bright Galactic bulge sources have luminosities of $L_{\mathbf{x}} \sim 10^{38} \mathrm{erg} \mathrm{s}^{-1}$, show X-ray flares lasting from minutes to hours, and often show a Z-type behavior on a color-color diagram (see later). The known orbital periods of these systems so far are $19 \mathrm{~h}$ (Sco X-1), 9.8 d (Cyg X-2), and $14 \mathrm{~d}$ (Sco $\mathrm{X}-2$ ), indicating that the secondaries in these systems are evolved. The evolution of the companion drives the mass transfer in these systems.

(ii) Lower-luminosity burst sources have $L_{\mathrm{x}} \sim 10^{36} \ldots 10^{37} \mathrm{erg} \mathrm{s}^{-1}$ (and are thus only 'lower-luminosity' compared to bulge sources, not compared to CVs!). These sources often emit sharp, bright bursts lasting seconds, and display atoll-shaped behavior in their color-color diagrams. Typical orbital periods in these systems range from $3 \ldots 15 \mathrm{~h}$, indicating 'normal' dwarf companions (although three have $P_{\text {orb }}<1 \mathrm{~h}$ and degenerate secondaries). In these systems it is magnetic braking and/or gravitational radiation that shrinks the orbit and drives the mass transfer. Dippers and transients are generally burst sources. All globular cluster LMXBs are burst sources.

My goal in this review is to supply a swift overview of various types of LMXB behavior for the non-expert, and in the process I will sometimes generalize without pointing out exceptions to the 'rules'.

\section{Orbital modulations}

At X-ray and optical energies, the appearance of the light curves from LMXBs and the existence of observable modulations depends strongly upon the source inclination. One empirical conclusion that we can draw very strongly from the data is that the accretion disks are not thin.

i $>\mathbf{8 0 ^ { \circ }}$. At high inclinations the central source is obscured by the disk edge, and the observed $\mathrm{X}$-rays are scattered through an accretion disk corona, producing a light curve with a smooth sinusoidal modulation. In 
addition to this modulation, we see a partial eclipse of the ADC by the companion. Modeling of the data from X1822-371, the canonical ADC system, shows that the corona must be extended such that it covers roughly the central third of the accretion disk. The optical light curve can be reproduced using a model with four components: the disk itself, its inner and outer rims, and the heated face of the secondary.

$\mathbf{7 5}^{\circ}<\mathbf{i}<\mathbf{8 0 ^ { \circ }}$. At these inclinations we $d o$ see the central source, although our view of it is frequently blocked by the companion star, producing sharp regular eclipses, as well as by azimuthal structure on the accretion disk, producing irregularly-shaped deep dips which lead the eclipses by $\sim 0.2$ in phase. The eclipses provide information about the size of the companion and the system geometry, and provide fiducial markers for timing studies of the orbital ephemeris and possible orbital period changes. The optical minima correspond to the times of X-ray eclipse. X0748-676 is a prime example of a source in this inclination range.

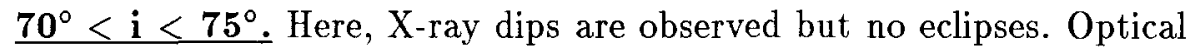
light curves become roughly sinusoidal, with minima occurring 0.2 in phase after the dips. Examples: X1254-690, X1916-053.

$\underline{\mathbf{i}}<\mathbf{7 0 ^ { \circ }}$. At inclinations lower than $70^{\circ}$ we cease to see such interesting effects. At X-ray energies no eclipses or dips are observed, and an orbital modulation is rarely seen. In the optical, the light curves are generally sinusoidal (e.g. X1735-444, X1636-536), with the exception of those sources in which ellipsoidal variations are observed from giant companions.

From these observations, an empirical model of a LMXB can be built up with all its important regions fixed in the binary's rest frame. If $\phi=0$ is the phase at which the NS is 'behind' the secondary (i.e. furthest from the observer), then eclipses of the central source (or partial eclipses by of the ADC) occur at this phase, and the accretion stream from the companion strikes the disk at $\phi=0.8 \ldots 0.85$, producing dips. Irregularly-shaped azimuthal structure associated with the impact point rises $\sim 15^{\circ}$ above the disk edge. Most of the DC optical emission comes from reprocessing in the disk, with an $\mathrm{AC}$ component from the heated face.

This will be a familiar picture for CV experts, as CVs also have strongly inclination-dependent modulations, sometimes show eclipses and dips, and often have activity associated with the stream impact point.

$\underline{X-r a y ~ d i p s ~ p r o v i d e ~ a ~ w e a l t h ~ o f ~ i n f o r m a t i o n ~ n o t ~ l i m i t e d ~ t o ~ t h e i r ~ r o l e ~ i n ~}$ defining the geometry of the system. From their irregular, ragged appearance and cycle-to-cycle differences in width and depth we know that the disk structure is dynamic. Also, spectral fitting results show that dips are associated with (but cannot be completely described by) increases in absorption 
of up to $N_{\mathrm{H}} \sim 10^{23} \mathrm{~cm}^{-2}$. (The dips at similar phases seen in a number of IPs are generally associated with much smaller $N_{\mathrm{H}}$ values $\lesssim 10^{21} \mathrm{~cm}^{-2}$.)

More complex and satisfying models show that spectra from dippers can be fit with a two-component model where one (the 'absorbed' component) is observed directly from the central source, obscured by the intervening material, and the other ('unabsorbed' component) is Thomson-scattered into the line of sight. The decrease in the normalization of the absorbed component relative to its value in quiescence (i.e. the non-dipping, non-bursting state) provides an estimate of the electron density of the absorbing medium, which can be combined with $N_{\mathrm{H}}$ to give a measure of the metal abundance of the material, assuming no photoionization occurs; such estimates show a metal underabundance of 1.6...2.5 in X1916-053, 2..7 in X0748-676, and up to 600 in X1755-338.

While the empirical 'model' of azimuthal structure seems secure, it poses difficulties for more theoretical models, which show that it is difficult to sustain the necessary vertical structure in a physically realistic way.

\section{X-ray bursts}

Type I X-ray bursts are caused by the thermonuclear detonation of material accreted onto the surface layers of the neutron star. In some accretion regimes, the nuclear burning in this material is unstable, and the catastrophic ignition and combustion of this material shows up observationally as a powerful burst from the system with a rise time of $\sim 1 \mathrm{~s}$ and a decay time of $\sim 10 \mathrm{~s}$. Bursts can be spectrally modeled using variations on a theme of blackbody emission. There are many differences in the details of bursts from different sources, and even from the same source: some bursts show photospheric radius expansion, which allows us (in principle) to measure both the mass and radius of the NS, directly examining the NS equation of state; double-peaked and triple-peaked bursts have been observed, some an apparent effect due to radius expansion, others a real effect due to variations in energy generation; and in bursts from some systems (X1608-522, $\mathrm{X} 1636-536, \mathrm{X} 1747-214)$ a transient absorption feature is observed in some bursts, perhaps due to a gravitationally red-shifted resonance line from the NS atmosphere.

Type II bursts are only seen in the Rapid Burster, and are caused by an as-yet poorly understood accretion instability mechanism. An excellent review of both types of X-ray burst behavior can be found in [4].

\section{X-ray spectroscopy: continuum and line emission}

Loosely speaking, the X-ray $(1 \ldots 20 \mathrm{keV})$ continuum spectrum from LMXBs can be defined as 'soft', with a characteristic thermal bremsstrahlung tem- 
perature of $k T \sim 5 \ldots 10 \mathrm{keV}$, as compared with the 'hard' spectrum of the MXRB pulsars, which have energy indices of $\alpha \sim 0 \ldots 1$.

In practice, higher-quality spectra show that for the brighter bulge $/ \mathrm{Z}$ sources a more complex two-component model is required, although there is some controversy about the spectral characterization and the physics underlying it [12]. The so-called 'Western model' consists of a Comptonized component from the scattering of low-energy photons on high-energy electrons, plus a $0.5 . .2 \mathrm{keV}$ blackbody which provides between 2 and $40 \%$ of the observed flux. Flares in the brighter sources can be modeled as an increase in this blackbody component, with the Comptonized component staying roughly constant. The 'Eastern model' to the same or similar data consists of a disk blackbody component, where the spectrum is built up by summing the blackbody contributions from an accretion disk in which the temperature varies as a function of radius $\left(T \propto r^{-3 / 4}\right)$, plus a $2 \ldots 3 \mathrm{keV}$ blackbody. In both cases the blackbody component is associated with the boundary layer between the disk and the neutron star.

For the lower-luminosity systems the continuum spectra can generally be fit with a power-law with energy index $\alpha=1$ and a high energy cutoff in the range $k T=5 \ldots 20 \mathrm{keV}$, although there are some exceptions; $\mathrm{X} 1820-303$ and X1608-522 have episodes in which a blackbody is required and the spectral behavior more closely resembles the bright bulge sources.

Iron $K$-line emission at $6.7 \mathrm{keV}$ is a feature of many LMXBs, including Sco X-1, Cyg X-2, and GX 5-1 as well as several fainter sources. In general this emission is broad, with a FWHM $\sim 0.5 \ldots 1 \mathrm{keV}$, but weak, with equivalent widths $(\mathrm{EW}) \sim 50 \ldots 100 \mathrm{eV}$. These lines have been resolved using EXOSAT GSPC, BBXRT, and now ASCA. At lower energies, line emission from $\mathrm{Fe} L, \mathrm{O}$ and $\mathrm{N} K$, and other species between 0.5 and $1.5 \mathrm{keV}$ have been detected with the Einstein OGS and SSS, and ASCA [9]. A spectacular new result from ASCA is the discovery of strong Neon Lyman $\alpha, \beta$ emission at $\sim 1.0 \mathrm{keV}$ from the compact degenerate LMXB X1626-67 [1], along with $\mathrm{O}$ Ly $\alpha$ and $\mathrm{He} \alpha$ at $0.65,0.58 \mathrm{keV}$. The data from the ASCA and Astro-E eras place severe demands upon the ongoing effort at many institutions to produce detailed theoretical plasma codes.

\section{Optical spectroscopy}

As seen from the NS, the accretion disk of the average LMXB subtends an angle of $\pm 10 \ldots 15^{\circ}$. Thus, the disk 'sees' about $25 \%$ of the source flux, or $\sim 10^{37} \mathrm{erg} \mathrm{s}^{-1}$, as compared to the $\sim 10^{33} \mathrm{erg} \mathrm{s}^{-1}$ produced by the M-dwarf companion, and so the optical continuum emission observed from LMXBs is dominated by reprocessing in the disk; the spectrum shows a bluish continuum, with an effective temperature of $\sim 310^{4} \mathrm{~K}$. The absolute magnitudes 
of LMXBs depends on the scale of the system; evolved systems such as Cyg X-2 have $M_{\mathrm{v}}=-2.0 \mathrm{mag}$, while other Z-sources have $M_{\mathrm{v}}=0 \mathrm{mag}$, bursters $M_{\mathrm{v}}=1 \ldots 2 \mathrm{mag}$, and the very short period systems (X1916-053, X1626-67, X1820-303, $\left.P_{\text {orb }}<1 \mathrm{~h}\right) M_{\mathrm{v}}=4 \ldots 5 \mathrm{mag}[11]$. Optical line emission from LMXBs is rather paltry compared to the spectacular riches that $\mathrm{CV}$ observers are used to. He II $\lambda 4686$ is generally the strongest line observed, with a mean strength of $\mathrm{EW}=5.5 \pm 3.8 \AA$ (the error represents the scatter across the class of LMXBs). The Bowen $\mathrm{C}$ III/N III blend at $\lambda \lambda 4640$ is also observed, with $\mathrm{EW}=2.8 \pm 2.1 \AA$, and sometimes emission from the Balmer series $(\mathrm{EW}(\mathrm{H} \beta)=2.6 \pm 3.7 \AA)$. The lines are formed variously in the heated face of the secondary, in the accretion disk, and/or in the accretion stream, depending upon the source.

Considering the energetics of the systems, the search for spectral features that may be associated with the secondary is a tricky proposition. The most detailed work to date has been done by Shahbaz et al. [7], who obtained spectra of a sample of 14 LMXBs between $4000 \ldots 9000 \AA$, and fit the spectra with combinations of standard accretion disk models plus companion star templates. Stellar features were detected only from systems with evolved companions (e.g. GX 1+4, Cyg X-2) and sources with small accretion rates (e.g. transients in quiescence Cen X-4, Aql X-1).

\section{Timing analysis}

\subsection{NEUTRON STAR SPIN PERIODS}

Pulsars are common in MXRB, and the Ritter catalogue [6] contains 19 CVs for which the white dwarf spin period is known, with $P_{\text {spin }}$ ranging from $28 \ldots 7500 \mathrm{~s}$. However, in LMXBs the pulsars appear to be a collection of exceptions to the rule rather than a separate class of objects in their own right.

Her X-1 $\left(P_{\text {orb }}=40.8 \mathrm{~h}, P_{\text {spin }}=1.24 \mathrm{~s}\right)$ shows a 35 -day high $/$ low cycle and optical brightness variations that march through the light curve every 35-day period, characteristics much more typical of an MXRB. X1627-67 $\left(P_{\text {orb }}=0.70 \mathrm{~h}, P_{\text {spin }}=7.7 \mathrm{~s}\right)$ has a degenerate companion in an ultra-compact binary. GX $1+4\left(P_{\text {spin }}=114 \mathrm{~s}\right)$ is a symbiotic system with an M6 III giant counterpart. The observed magnetic fields in Her X-1 and GX $1+4$ are 3 orders of magnitude higher than the 'normal' range for LMXBs. Each of these systems, then, probably has an evolutionary history that deviates from the norm.

For more 'standard' LMXBs the neutron star spin period is unknown. $\mathrm{X}$-ray burst theory constrains fields to $B \lesssim 10^{10} \mathrm{G}$, while horizontal branch QPO (see below) require $B \sim 10^{9} \mathrm{G}$. The expected NS spin period depends upon the accretion torques over the lifetime of the system, and physical 
models and observational results from radio pulsars and QPO suggest that NS spin periods should be in the range $1 \ldots 10 \mathrm{~ms}$. Sophisticated searches for these periods have been performed on a sample of the brightest LMXBs, including all the $\mathrm{Z}$-sources, to $512 \mathrm{~Hz}$, and have placed upper limits on the pulse fractions of $0.3 \ldots 5 \%$.

\subsection{Z/ATOLL SOURCES, QPO, AND NOISE}

Timing analysis has nevertheless proved crucial to interpreting LMXB behavior, through the study of quasi-periodic oscillations (QPO) and related noise phenomena. QPO are defined as peaked noise in a frequency-power plot, where the width of the peak is less than half of the peak frequency. (36 CVs also show QPO, but their time-scale and interpretation are rather different.)

A principal diagnostic tool is the color-color diagram, where the higherenergy hardness ratio is plotted against a lower-energy hardness ratio to produce a diagram similar in concept to the Hertzprung-Russell diagram. Six of the brighter LMXBs (GX 5-1, Cyg X-2, Sco X-1, GX 17+2, GX 340 +0 , GX 349-2=Sco X-2) describe a Z-shaped track on such a diagram [2]. Sources move along the $\mathrm{Z}$ continuously, typically tracing out a complete $Z$ in $\sim$ days, rather than jumping tracks from one state to another. The $Z$-shape persists from year to year, although some evidence for a secular shift in the position of the entire $\mathrm{Z}$ is seen in Cyg X-2 [3].

Fig. 1 shows an example of such a Z-shaped curve, with the Horizontal, Normal, and Flaring branches (HB, NB, FB) labeled. Mass transfer increases in the direction shown by the arrow. In the HB, QPO are observed with a peak frequency $F_{\text {peak }}$ of $13 \ldots 55 \mathrm{~Hz}$, with $F_{\text {peak }}$ increasing with $\dot{M}$. NB/FB QPO appear at a lower frequency range of $6 \ldots 20 \mathrm{~Hz} ; \mathrm{HB}$ and NB QPO are sometimes observed simultaneously, indicating that they have different physical origins.

As the lower left portion of the figure shows, substantial noise components are also seen from the $Z$ sources: high frequency noise (HFN), with a cutoff energy of $50 \ldots 100 \mathrm{~Hz}$; low frequency noise (LFN) at $2 \ldots 20 \mathrm{~Hz}$ (seen only on the HB), and very low frequency noise (VLFN) that appears most strongly in the flaring branch, with a power index of $\alpha=1.5 \ldots 2.0$.

Other sources, including some bright bulge sources as well as all the bursters, show a different pattern in the color-color diagram, resembling an atoll divided into three areas: Upper Banana, Lower Banana, and Island (UB, LB, IS). Atoll sources do not display QPO, although they do show noise characteristics; HFN is seen extending from $1 \ldots 100 \mathrm{~Hz}$, occasionally with a broad band-limited peak that mimics (but is different from) QPO behavior. VLFN is also seen in the atoll sources. 

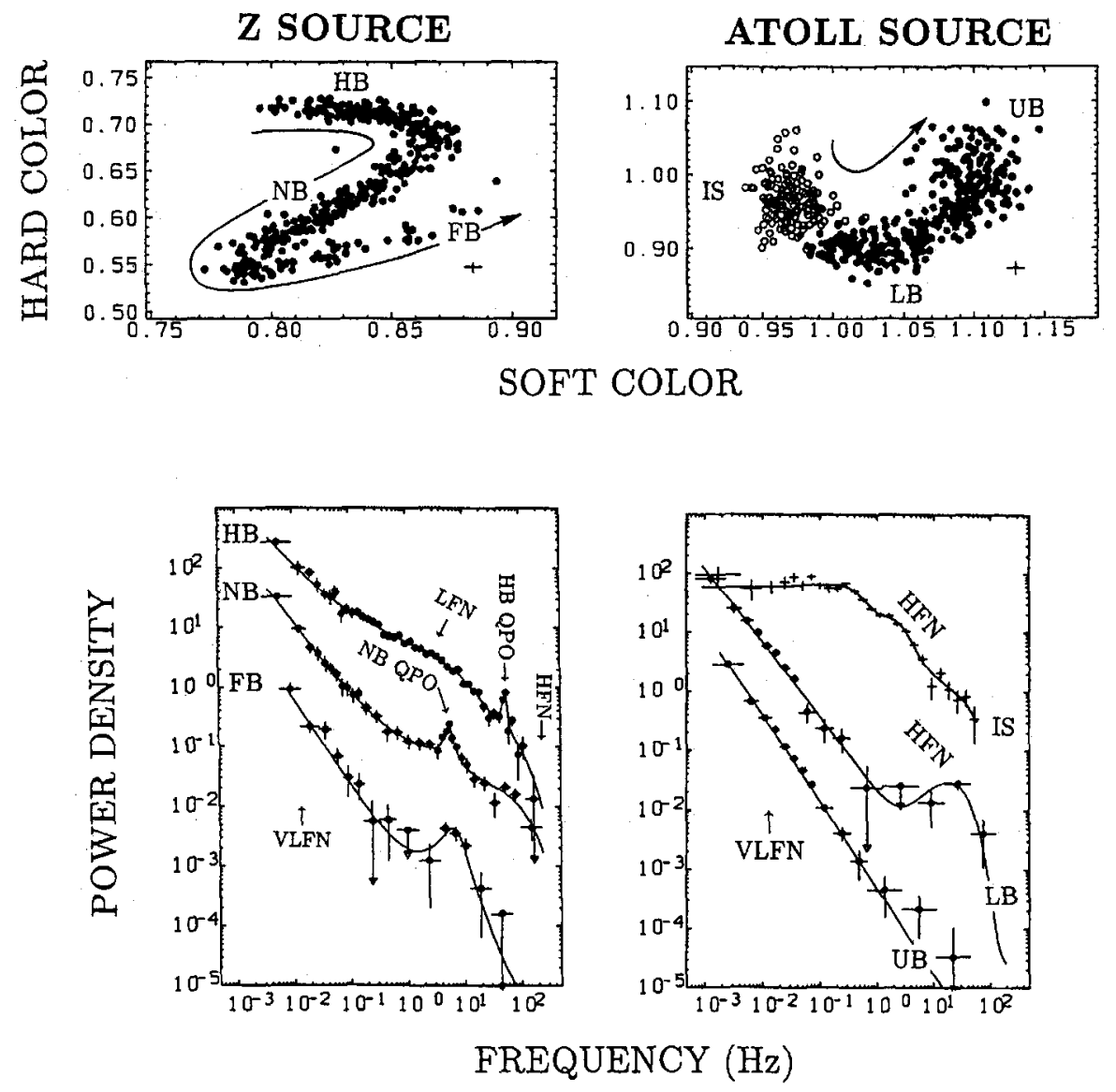

Figure 1. The behavior of $\mathrm{Z}$ and atoll sources in the color-color diagram, and in frequency space [10]. (Figure provided courtesy of Michiel van der Klis.)

HB QPO are normally interpreted in terms of the beat frequency model. Plasma at the inner edge of the accretion disk interacts with the magnetosphere of the rapidly-spinning neutron star at the beat frequency between the Keplerian velocity of the inner disk material and the NS spin frequency. This model gives an expected $P_{\text {spin }}$ in the range $3 \ldots 20 \mathrm{~ms}$, which is in line with the predictions given in the previous section.

Models suggest that the HB covers accretion rates between $0.5 \ldots 0.9$ $\dot{M}_{\text {Edd. }}$ As the accretion rate rises above this, radiation pressure becomes critical in the inner disk and the system approaches spherical accretion. The NB QPO seen in this regime may be caused by oscillations in the accretion flow, perhaps due to feedback between X-ray production and accretion. As 
the source goes over $\dot{M}_{\text {Edd }}$ and into the FB, even this radial inflow becomes unstable and non-radial oscillation modes may become important. VLFN may be due to either the time-scale on which the source moves along the $\mathrm{Z}$, or the time-scale of convective burning fronts on the NS surface.

Based on the ideas above a simple, consistent physical model has been proposed [5]. In this model, the Z sources contain NS with magnetic fields $B \sim 10^{9} \mathrm{G}$, which generate soft photons by high-harmonic cyclotron emission. These photons are Comptonized by electrons in a hot central corona (HCC) around the $30 \mathrm{~km}$ radius magnetosphere, producing a flat spectrum with a cutoff energy equivalent to the electron temperature $(\sim 10 \mathrm{keV})$. From this beginning, numerical models reproduce the spectral changes observed as the mass accretion rate increases, including the Z-shaped tracks on the color-color diagram. The same model can also generate the behavior seen from atoll sources, if the magnetic field is an order of magnitude weaker and the NS accretes at a much lower rate of $0.01 \ldots 0.1 \dot{M}_{\text {Edd }}$.

The emerging picture of LMXB behavior has some pleasing consistencies. Pulsars show noise spectra similar to the band-limited HFN at $10^{2} \mathrm{~Hz}$ seen in Z-sources, supporting the idea that this noise originates near the magnetosphere. Atoll sources share noise characteristics at $1 \ldots 10 \mathrm{~Hz}$ with both Z-sources and black hole candidates, supporting the origin of this noise in the inner (radiation-dominated) disk. Thus, a basic unified picture of timing and spectral behavior in all the $\mathrm{X}$-ray binaries requires just two structures: the magnetosphere, and the inner disk region.

\subsection{LONGER-TERM TIMING PHENOMENA: CYCLES AND ORBITAL PERIOD CHANGES}

In MXRBs firm evidence exists for long-term cycles from 30...300 d in LMC $\mathrm{X}-4$, SS 433 , LMC X-3, Cyg X-1, and Her X-1, probably due to the changing aspect of a tilted, twisted accretion disk, sometimes in retrograde precession. In LMXBs long-term cycles are rare and have been confirmed in only two systems, X1820-303 $\left(P_{\text {long }}=175 \mathrm{~d}\right)$ and X1916-053 $\left(P_{\text {long }}=199 \mathrm{~d}\right)$. In these systems disk precession is unlikely, and the generally-accepted model involves intrinsic variations in mass transfer. One possibility is that the mass transfer events are analogous to SU UMa-type superoutbursts, for which the bright spot luminosity also implies enhanced mass transfer with recurrence rates of $130 \ldots 400 \mathrm{~d}$.

Three LMXBs (X1820-303, X0748-676, X1822-371) show orbital period changes either greatly in excess of what would be expected through evolutionary models, or with the wrong sign. Some possible physical causes are: $\mathrm{X}$-ray heating of the companion, causing mass loss, radius changes, and/or simulated wind accretion; stellar magnetic activity; hierarchical triple systems, modulating the orbital period derivations by Keplerian motion; and 
solar-type cycles in the companion which alter its radius and therefore the orbital period. The last of these explanations has been invoked in CVs, $\sim 20$ of which show some evidence for $P_{\text {long }}=1 \ldots 20 \mathrm{yr}$. A review of precession and other long-term cyclic behavior in X-ray binaries can be found in [8].

\section{Conclusions}

A few key parameters define the observed phenomenology of both LMXBs and CVs. The differences in behavior between subclasses of each source type largely result from differences in: (i) accretion rate; (ii) mass ratio of components; (iii) magnetic field strengths, and the existence and location of the magnetosphere; (iv) source inclination, and the characteristics, extent and edge structure of disks; and (v) X-ray heating.

The phenomenology of LMXBs now seems to be well understood, at least of time-scales of $\sim$ days down to $\sim$ a few ms. The $\mathrm{Z}$-sources display QPO and various noise characteristics, have higher accretion rates (0.6 $\left.\ldots 1.1 \dot{M}_{\text {Edd }}\right)$ and higher magnetic field strengths $\left(B \sim 10^{9} \ldots 10^{10} \mathrm{G}\right)$, and show flaring behavior and two-component spectra. The atoll sources have lower accretion rates $\left(0.01 \ldots 0.1 \dot{M}_{\text {Edd }}\right)$ and lower magnetic field strengths ( $B \sim 10^{8} \ldots 10^{9} \mathrm{G}$ ), and frequently (but not always) show Type I bursts and single-component continuum spectra. Iron line emission at $6.7 \mathrm{keV}$ and other line features at lower energies are observed in both classes. Dips and bursts are predominantly seen in the atoll sources.

\section{References}

1. Angelini, L., White, N.E., Nagase, F., et al., 1995, Ap. J., 449, L41

2. Hasinger, G., van der Klis, M., 1989,, A\&A, 225, 79

3. Kuulkers, E., van der Klis, M., Vaughan, B., 1996, A\&A, in press

4. Lewin, W.H.G., van Paradijs, J., Taam, R.E., 1993, Spa. Sci. Rev., 62, 223

5. Psaltis, D., Lamb, F.K., Miller, G.S., 1995, Ap. J. Lett., 454, L137

6. Ritter, H., Kolb, U., 1995, in "X-ray Binaries", eds. Lewin, W.H.G., van Paradijs, J. and van den Heuvel, E.P.J., CUP, p578

7. Shahbaz, T., et al., 1995, in preparation; Shahbaz, T., Ph.D. thesis, U. Keele.

8. Smale, A.P., 1994, in "The Evolution of X-ray Binaries", eds. S.S. Holt and C.S. Day, AIP, New York, p525

9. Smale, A.P., White, N.E., Angelini, L., et al., 1996, in preparation.

10. van der Klis, M., 1994, Ap. J. Supp., 92, 511

11. van Paradijs, J., McClintock, J.E., 1995, in "X-ray Binaries", eds. Lewin, W.H.G., van Paradijs, J., van den Heuvel, E.P.J., CUP, p58

12. White, N.E., Stella, L., Parmar, A.N., 1988, Ap. J., 324, 363

Due to space constraints I have only supplied references to other reviews and to papers that are pivotal, very recent, or unusually obscure. 